\title{
Nineteenth and Twentieth Century Clouds Over the Twenty-First Century Virtual Observatory
}

\author{
P. J. E. Peebles \\ Joseph Henry Laboratories, Jadwin Hall \\ Princeton University, Princeton NJ 08544 USA
}

\begin{abstract}
Physical science has changed in the century since Lord Kelvin's celebrated essay on Nineteenth Century Clouds over the Dynamical Theory of Heat and Light, but some things are the same. Analogs in what was happening in physics then and what is happening in astronomy today serve to remind us why we can be confident the Virtual Observatory of the twenty-first century will have a rich list of challenges to explore.
\end{abstract}

\section{Introduction}

Astronomy has enjoyed a very good century. Have the basic problems now been solved, leaving for the astronomers of the $21^{\text {st }}$ century the task of working out the pesky details? The question is little discussed - astronomers are too busy with ongoing research - but worth considering from time to time. I shall argue that we have a useful guide to the long-term prospects for research in astronomy from analogs to the present situation in was happening in physics 100 years ago. In both cases there is a basis of fundamental concepts that are strikingly successful, apart from some stubborn clouds, or, as we would now say, challenges for research. The clouds over electromagnetism and thermal physics at the start of the $20^{\text {th }}$ century foreshadowed relativity and quantum physics. We can't say what will be learned from the clouds over present-day astronomy - I shall mention aspects of the dark sector, strong space curvature, and the meaning of life but we can be sure they will continue to drive difficult but fascinating research in astronomy for quite some time to come.

\section{Physics at the Start of the $20^{\text {th }}$ Century}

The elements of the situation in physics a century ago have been retold to generations of students, and rightly so; these are golden moments in the history of physical science. And I think they are an edifying example for our assessment of the present state of research in astronomy.

At the start of the $20^{\text {th }}$ century physicists had good reason to believe they had securely established laws of electromagnetism and thermal physics, well tested in the laboratory and applied in rapidly growing power and communications industries; Lord Kelvin's fortune came from his contributions to the design of the transatlantic telegraph cable. But he and others were well aware of flaws, or clouds, in the physics, as famously summarized in Kelvin's [1] essay in 1901. 
Kelvin's Cloud No. I is the luminiferous ether. The experimental situation is also discussed in Lecture VIII, The Ether, in Michelson's Light Waves and Their Uses [2]. You can read about the familiar experiments - Michelson's discovery of the isotropy of the velocity of light, and the Fizeau measurement of the addition of velocities of light and fluid in a moving fluid - and others that are less celebrated but as remarkable. My favorite among the latter is the measurement of annual aberration in a telescope that is filled with water so as to reduce the velocity of light. The results are no surprise to us, but a real problem for Kelvin and Michelson. Kelvin mentions with approval the contraction idea of Fitzgerald and Lorentz, but concludes "I am afraid we must still regard Cloud No. I as very dense" [1]. Einstein's brilliant insight cleared the cloud, and gave us special relativity theory. If that had not happened I have to believe people would soon have pieced together the full theory from these remarkable measurements.

Kelvin's Cloud II is the inconsistency of the law of partition of energy at thermal equilibrium with the measured ratios $C_{p} / C_{v}$ of heat capacities of gases at constant pressure and volume.[Kelvin [1] quotes Rayleigh's assessment [3]: "The difficulties connected with the application of the law of equal partition of energy to actual gases have long been felt. In the case of argon and helium and mercury vapour the ratio of specific heats (1.67) limits the degrees of freedom of each molecule to the three required for translatory motion. The value (1.4) applicable to the principal diatomic gases gives room for three kinds of translation and for two kinds of rotation. Nothing is left for rotation round the line joining the atoms, nor for relative motion of the atoms in this line. Even if we regard the atoms as mere points, whose rotation means nothing, there must still exist energy of the last-mentioned kind, and its amount (according to the law) should not be inferior." Something certainly is wrong. Kelvin accepted the mechanics and questioned the assumption of strict statistical equilibrium. Planck (1900) hit on the fix, to the mechanics, in the model for blackbody radiation, and Einstein (1907) applied the fix to heat capacities, in early steps to quantum physics.

It is often said, at least in introductory remarks in courses on modern physics, that people were a lot more impressed by the successes of physics in 1900 than by the clouds, that the feeling was that physics is essentially complete, apart from fixing a few problems and adding decimal places. The famous example is Michelson's statement (in [2], p.23), that the "more important fundamental laws and facts of physical science have all been discovered, and these are now so firmly established that the possibility of their ever being supplanted in consequence of new discoveries is exceedingly remote." This is clear enough, and Badash [4]

\footnotetext{
1 This memorable story has been taught to generations of students in the introduction to quantum mechanics, some of whom I hope actually appreciated it. Equipartition in classical mechanics says that at thermal equilibrium at temperature $T$ the mean energy belonging to each quadratic term in the Lagrangian is $k T / 2$. It follows that if each atom or molecule in a gas has $\nu$ quadratic terms associated with its internal structure then, taking account of the $p d V$ work at constant $p$, the ratio of heat capacities is $C_{p} / C_{v}=(5+\nu) /(3+\nu)$. Thus classical physics predicts that a gas of point-like particles has $C_{p} / C_{v}=5 / 3$, and a gas of atoms with a rich internal structure, so $\nu$ is large, has $C_{p} / C_{v}$ close to unity.
} 
shows Michelson repeated these sentiments elsewhere, so at the time he must have meant it. But consider Michelson's summary statement in the same book, at the end of the chapter on the ether ([2], p. 163): "The phenomenon of the aberration of the fixed stars can be accounted for on the hypothesis that the ether does not partake of the Earth's motion in its revolution about the sun. All experiments for testing this hypothesis have, however, given negative results, so that the theory may still be said to be in an unsatisfactory condition." And earlier in the summary he says "Little as we know about it [the ether], we may say that our ignorance of ordinary matter is still greater." Here Michelson sounds like someone who sees very real challenges.

These challenges drove hard work, as in Fizeau's remarkable waterworks and Michelson's [2] massive arrangements to suppress vibrations: "the apparatus was mounted on a stone support, about four feet square and one foot thick, and this stone was mounted on a circular disc of wood which floated in a tank of mercury." I see no evidence of complacency in Kelvin's ([1], p. 17) struggle to visualize mercury vapor atoms, which are capable of producing a rich line spectrum but at thermal equilibrium in laboratory conditions seem to be incapable even of rotating, or in Rowland's 1899 presidential address to the American Physical Society [5: "What is matter; what is gravitation; what is ether and the radiation through it; what is electricity and magnetism; how are these connected together and what is their relation to heat? These are the greater problems of the universe. But many infinitely smaller problems we must attack and solve before we can even guess at the solution of the greater ones."

Badash [4] gives a valuable survey of opinions across a broader range of the academic community, and concludes that at the end of the $19^{\text {th }}$ century the idea that science is reaching completeness 'was more a "low-grade infection," but nevertheless very real.' This sounds right, but my impression is that the infection had little effect on the research of leading physicists, including Michelson.

The confidence in the established parts of physics at the start of the $20^{\text {th }}$ century was well placed: we still use and teach this electromagnetism and thermodynamics - though we now think of it as part of a hierarchy of approximations that for all we will ever know may run arbitrarily deep. Concerns about the $19^{\text {th }}$ clouds could not have anticipated the vast enlargement of physics and our worldview in the $20^{\text {th }}$ century, but the point for our purpose is that the clouds were recognized and driving research.

I offer some parallels to the situation in present-day astronomy. We know how stars like the Sun shine, but there are big gaps in our understanding of how stars form, at high redshift and even in our own galaxy. I classify star formation as a Rowland-type "smaller problem:" it is fiendishly difficult but approachable by well-motivated lines of research involving standard physics (as far as we know). Such Rowland-type problems are the key to a healthy science, and astronomy has them in abundance. We know the universe is evolving, and the evidence is that general relativity theory gives a good description of the dynamics. But we don't know what the universe is made of - apart from the five percent or so in the visible sector - or what happens when spacetime curvature gets large, 
as was the case in the very early universe and happens now in the centers of galaxies. These are Kelvin-level clouds: critical issues whose resolution would greatly advance our understanding of the material world. We don't know what the present-day clouds are hiding, but we can be sure they will continue to be a good focus for research.

\section{Astronomy at the Start of the $21^{\text {st }}$ Century}

The situation in astronomy in 1900 was close to the academic myth about physics. Badash [4] gives a good quote from Newcomb [6]: "we do appear to be fast approaching the limits of our knowledge ... one comet is so much like another that we cannot regard one as adding in any important degree to our knowledge. The result is that the work which really occupies the attention of the astronomer is less the discovery of new things than the elaboration of those already known, and the entire systemization of our knowledge." The main systemization was the cataloging of angular positions, apparent magnitudes, and spectral classifications of literally hundreds of thousands of stars. But this dreary labor led to wonderful new things; consider these two examples of research trajectories.2

Eddington's (1924) gas spheres gave Bethe (1938) the physical conditions for nuclear reactions in stars, and a way out of the discrepancy between the Helmholz-Kelvin (1860) Solar cooling time and the much greater geological times from radioactive decay ages. A beautiful recent development is the demonstration that the Solar neutrino luminosity really is in satisfactory agreement with the theory of the Solar nuclear reaction rates, to be understood with the help of the demonstration of nonzero neutrino masses.

Kapteyn (1901) set the distance scale for star counts in our island universe, Shapley (1918) enlarged the island, and Hubble (1925) placed it in the near homogeneous realm of the nebulae. Hubble's linear relation between his distances to the nebulae and Slipher's (1914) redshifts led Lemaitre (1927) to the now standard model for the expanding universe. The most direct evidence that our universe actually is evolving - expanding and cooling - was completed with the demonstration by the USA COBE and Canadian UBC experiments (1990) that the $3 \mathrm{~K}$ cosmic background radiation spectrum is very close to thermal. In the 1930s Hubble commenced the great program of cosmological tests to check the relativistic Friedmann-Lemaitre model for the expanding universe. Now, seven decades later, we are approaching a satisfactory application of the tests, which the relativistic cosmology passes so far.

\footnotetext{
${ }^{2}$ I have taken the liberty of indicating contributions by several people, and even groups, under the name of a representative leading figure, with an approximate year for developments that in some cases occurred over many years. I hope it is understood that another reviewer could choose very different representative examples of what happened in $20^{\text {th }}$ century astronomy. Harwit [i] presents a well-documented and much more complete analysis of discoveries in astronomy and the prospects for discoveries of new astronomical phenomena.
} 
A byproduct of the cosmological tests is evidence that structure grew out of a mass distribution at high redshift that is specified by one function of one variable, the near scale-invariant power spectrum of a random Gaussian process. There are problems with details, as will be discussed, but the evidence pretty strongly indicates this is a good approximation to the way it is. The Rowlandtype problem, of breathtaking scope and complexity, is to demonstrate that standard physics actually can account for the origin of the worlds and their spectacular variety of phenomena out of this simple initial condition.

I have mentioned stories with some happy endings, in reasonably conclusive resolutions of lines of research that have occupied generations of astronomers. We cannot say whether more happy endings to big puzzles are in store, but we get some feeling for the prospects by considering present-day clouds over astronomy. I shall comment on two from the $20^{\text {th }}$ century and one from the $19^{\text {th }}$ century.

\subsection{Cloud No. I: the Dark Sector}

The dark sector includes the nonbaryonic matter that is thought to dominate the outer parts of galaxies and clusters of galaxies; Einstein's cosmological constant, $\Lambda$, or dark energy that acts like it; and the vacuum energy density. The darkest part of the cloud is over the vacuum energy. I draw these comments from a review of the issues in [8] and the executive summary in [9].

Nernst 10 seems to be the first to have discussed the energy of the quantum vacuum, in 1916. His zero-point energy for each mode of oscillation of the electromagnetic field is off by a factor of two, remarkably good considering this was before Heisenberg and Schrödinger. Nernst showed that the sum over zero-point energies of the modes with laboratory wavelengths is on the order of $1 \mathrm{~g} \mathrm{~cm}^{-2}$. Pauli (in [11], p. 250) was quite aware that this mass density would be ruinous for relativistic cosmology; he advised that we just ignore the zero-point energy of the electromagnetic field. This is a prescription, of course, and not even a rational one. Pauli certainly knew that one must take account of zero-point energies to get the right binding energies in nonrelativistic particle mechanics. We now know the same applies to gravitational masses. And in standard physics the zero-point energies of fields are just as real. The problem with the vacuum energy density has persisted - if anything grown more puzzling - through all the spectacular advances in physics in the $20^{\text {th }}$ century. I like Wilczek's phrase: this aspect of our physics is "profoundly incomplete" [12]. It is a Kelvin-level cloud: within physics that is wonderfully well tested and successful in a broad range of applications there is a distinct glitch.

We have observational probes that might be helpful. If the vacuum presents the same properties to any inertial observer, its effect on spacetime curvature is the same as Einstein's cosmological constant, $\Lambda$. The evidence from the cosmological tests is that the expansion of the universe actually is dominated a term that acts like $\Lambda$ - though the absolute value is ridiculously small compared to what is suggested by current ideas in particle physics. The case for detection is serious, but since it depends on difficult observations and insecure models I 
am inclined to limit the odds to maybe five to one. But work in progress should convincingly show us whether a term that acts like $\Lambda$ really is present.

Until recently the tendency in the astronomy community has been to hope that it could get by with Pauli's prescription, or at worse the phenomenological description of the vacuum by the numerical value of one constant, $\Lambda$, leaving the dispersal of this cloud to the physicists. But current ideas are that $\Lambda$ is only an approximation to a dynamical entity, dark energy, whose mass density varies with time on the scale of cosmic evolution, and varies with position in response to the large-scale irregularities in the matter distribution. Detection of these effects would not solve the vacuum energy density problem, but it would be a spectacularly stimulating clue. We know how it might be done, and I have been hearing ambitious plans to make the astronomical measurements. You may be sure the physicists will be hanging on every word of progress; they are desperate for something to knock them off dead center.

In the standard cosmology the dark sector also contains nonbaryonic matter that dominates the mass in the outer dark halos of galaxies and the mass in clusters of galaxies. I am in sympathy with those who ask for more evidence this nonbaryonic matter really exists, but I think the case already is close to compelling. The clearest exhibition of dark matter is the giant luminous arcs the gravitationally lensed images of background galaxies produced by the gravitational deflection of light by the masses in clusters of galaxies. No force law I can imagine could produce these smooth arcs out of gravitating matter with the clumpy distribution of the starlight in clusters. There has to be cluster dark matter, and if it were baryonic it would cause ugly problems [8].

We have little empirical guidance to the physics of the dark sector: we are working in the dark. We accordingly adopt the simplest physics we can get away with, which is good strategy, but certainly need not be the whole story: consider that polytropic ideal gas spheres were good enough for Eddington's analysis of the structure of the Sun, but helioseismology reveals a host of new details. If our model for the dark sector is missing details that matter it will be revealed by problems in fitting the observations. And there are hints of problems, from observations of the structure and formation of galaxies. My list is headed by the prediction that elliptical galaxies form by mergers at modest redshifts, which seems to be at odds with the observation of massive quasars at $z \sim 6$; the prediction of appreciable debris in the voids defined by $L_{*}$ galaxies, which seems to be at odds with the observation that dwarf, irregular, and $L_{*}$ galaxies share quite similar distributions; and the prediction of cusp-like dark matter cores in low surface brightness galaxies, which is at odds with what is observed. These are Rowland-type problems that draw on the rich phenomenology of astronomy, from the latest observations by the Hubble Space Telescope to the vast accumulation of lore from decades past. Sorting through all this takes time, but I expect will show us whether the problems with the standard picture for the dark sector will be resolved by better understanding of the observations and theory, or will be promoted to a Kelvin-level cloud. 


\subsection{Cloud No. II: Strong Spacetime Curvature}

Cloud II is the singularities of general relativity, where the theory becomes meaningless. It took some time for people to sort out the physical singularities from singular coordinate labels, and to face up to the phenomenological importance of the former. I remember as a graduate student in the late 1950s reading a distinguished physicist's elegant picture of the bounce in an oscillating universe: like turning a glove inside out, one finger at a time. In the mid 1960s Penrose's 13 pioneering approach to singularity theorems forced us to accept that we need deeper physics to see past the formal singularity at infinite redshift in the relativistic Friedmann-Lemaitre cosmological model. At about the same time, the discovery of quasars, and the broader recognition of active galactic nuclei, offered an example of strong spacetime curvature in compact objects closer to hand. Now, a half century later, we have rich phenomenologies of compact objects and cosmology, and we still have the singularities.

Analyses of the astrophysics of massive compact objects - those observed at the centers of large galaxies, and star remnants more massive than a white dwarf - usually take as given a Schwarzschild or Kerr black hole geometry with a truly black inside, in discussions of what have grown to be quite detailed observations. There are no problems with this approach, a sign of the remarkable predictive power of general relativity theory. But good science demands that we seek positive evidence in support of the black hole picture, and watch for credible evidence that the standard picture may not be quite right. Maybe advances in fundamental physics will show us what really is happening in the centers of galaxies, or maybe the dispersal of this cloud will be guided by the phenomenology.

Analyses of observations in cosmology finesse the formal singularity of the Friedmann-Lemaitre model, and the unknown physics at the Planck scale, by stipulating initial conditions at a more modest redshift, let us say $z=10^{15}$. Nowadays the initial conditions often are given a pedigree, from the inflation model, and the observational constraints on the initial conditions are used to infer conditions on what was happening during inflation. But, since the inflation scenario can fit a considerable range and variety of initial conditions, we don't know whether these measures of the very early universe amount to anything more than a "just so" story. Three assignments may help.

We look to observational astronomers and cosmologists for tighter constraints on the initial condition at redshift $z=10^{15}$. And it behooves us to watch for hints that there is more to learn about cosmic evolution than is encoded in this initial condition within the present standard cosmology. The successes of the extrapolation of standard physics to the length and time scales of cosmology are impressive, but the enormous extrapolation certainly allows room for surprises. I am watching for them in the problems with galaxy formation I mentioned in connection with the dark sector.

We look to those exploring ideas about the early universe to try to find alternatives to inflation. If all due diligence yielded none we would have an argument by default that inflation really happened, a dismal closure but better than nothing. Alternatives are under discussion; it will be of great interest to 
know whether some variant of the ekpyrotic universe [14 has a physical basis comparable to that of inflation, which is not asking all that much.

We look to the physics community to build a firmer basis for cosmology at high redshift. If fundamental physics converged on a complete theory that predicts a definite version of inflation, or some other picture for the early universe, which agrees with the astronomical constraints, it will convincingly complete cosmology. The prediction's the thing, of course.

\subsection{Cloud No. III: the Meaning of Life}

This is a cloud over a much broader community. We can leave to the experts in other fields the philosophical issues, and the analysis of the molecular basis for life. The task for astronomy and its Virtual Observatory is to search for evidence of extraterrestrial life. This is a Kelvin-level cloud: a powerful driver of research whose outcome could profoundly affect our worldview.

Maybe life on Earth came from primitive extraterrestrial seeds; Hoyle and Wickramasinghe 15] survey the history and present state of ideas. Maybe there are advanced forms of life on other worlds, seeded or evolved out of spontaneously created life. The familiar $19^{\text {th }}$ century example of the search for organized life is Lowell's study of possible signs on Mars; the search continues in the SETI and OSETI projects. The Terrestrial Planet Finder (TPF) will search for Earth-like worlds where life might flourish in a primitive or organized state.

I read that the search for extraterrestrial life is the part of astronomy that most interests most people. I offer four observations of how the big ideas and activities in society have influenced the directions of this research.

First, Charles Darwin's deeply influential arguments for evolution by natural selection forced debate on what the first step in the evolution of life might have been. At about the same time, people were coming to the conclusion that spontaneous generation is an exceedingly rare event, if it happens at all, and maybe contrary to Darwin's principle that life evolves out of life [16, 17]. It was natural therefore that people turned to the idea of extraterrestrial seeds. Helmholtz (1874), a most influential physicist and physiologist, argued for the idea, as did an important chemist, Arrhenius (1908). Kelvin (1871) endorsed the general idea, but not natural selection: he argued for "intelligent and benevolent design" 18 .

Second, the end of the $19^{\text {th }}$ century was a time of large-scale civil engineering, including completion of the modern Suez Canal in 1869. It is perhaps not so surprising that Lowell looked for signs of big engineering on Mars.

Third, this is an age of computers and information transfer. I think it's not surprising that people are searching for extraterrestrial bar codes. I don't mean to mock serious and important science: a source of bar codes would signify self-aware life by any definition. Imagine the effect on our society of the demonstration that there actually is extraterrestrial self-aware life, that might even have something to say to us.

Fourth, this is an age of big science, that is supported by the wealth of nations. A logical consequence is that research in science is influenced by big gov- 
ernment. The TPF is a recent example: this is pure curiosity-driven big science that originated within government funding agencies, rather than being forced by intense pressure from a scientific community.

I offer two lessons from these observations. First, the fascination with the idea of life on other worlds has a long history, back through the $19^{\text {th }}$ century, and, I expect, it has a long future. But societies evolve, and it is natural to expect the focus of the search for extraterrestrial life will evolve too.

Second, the means of support of the scientific enterprise are evolving; the TPF is leading the curve. The TPF certainly may yield wonderful results; we have the inspiring precedent of Slipher's discovery of the cosmological redshift, at the observatory Lowell built with a goal paralleling that of the TPF. But there is the difference that funding agencies have to tend to many masters; they can't have the compulsive attention span of curiosity-driven people like Lowell. The Virtual Observatory is not leading this curve: a community is fighting for it, in the style of what gave us the space telescope, and what happened in physics in the last half century. These are generally happy examples - apart from such glitches as sunset clauses - of what I suppose is an inevitable development: the directions of research in astronomy are increasingly influenced by government as well as society, and astronomers must continue learning how to deal with it.

\section{Concluding Remarks}

Our ability to explore the physical universe is limited by resources and intellectual energy: the scientific enterprise must eventually reach completion by exhaustion. But we can be sure this will not happen any time soon to astronomy and its Virtual Observatory, because the subject has a rich list of Rowlandtype problems to address, and, as I have discussed, a key role to play in the exploration of clear and present Kelvin-level gaps in our understanding of the fundamental basis for physical science. There was no guarantee in 1900 that the clouds over physics would clear, with a wonderful expansion of our knowledge. It would be foolish to try to guess what the present clouds might foreshadow, but we can list the general possibilities. Maybe the clouds will resist all efforts at resolution. If so, convincing people of this certainly will generate a lot of work for astronomers. Maybe the clouds will be cleared and at last leave astronomers to tidy up the pesky details. Or maybe clearing the clouds will reveal a new set, as has happened before.

I have avoided until now commenting on a serious issue under debate in the astronomy community: is this an appropriate time to commit limited resources to an International Virtual Observatory? I respect the arguments against, but am persuaded by personal experience that the growth of the Virtual Observatory is inevitable and would benefit from intelligent design. Two years ago the walls of my office were covered by about 25 meters of journal rows, dating back to 1965. I loved the convenience of reaching for a copy of the wanted article. But I've discarded the journals; I love even more the much greater convenience and power of ADS, arXiv, and JSTOR. I notice many colleagues feel the same: we have 
become addicted to these Virtual Libraries. Present-day Virtual Observatories are a useful but limited counterpart. Their further development seems to me to be an inevitable part of what we see happening around us, and surely calls for the proactive community response I have observed at this meeting.

\section{Acknowledgments}

I have benefitted from advice from Larry Badash, Neta Bahcall, Jeremy Bernstein, Masataka Fukugita, Rich Gott, Martin Harwit, Gerald Holton, Stacey McGaugh, Bharat Ratra, Paul Schechter, Max Tegmark, and Ed Turner. This work was supported in part by the USA National Science Foundation.

\section{References}

1. Lord Kelvin: Phil. Mag. ii - sixth series, 1 (1901)

2. A. A. Michelson: Light Waves and Their Uses (University of Chicago Press, Chicago 1903)

3. Lord Rayleigh: Phil Mag. xlix - fifth series, 98 (1900)

4. L. Badash: Isis 63, 48 (1972)

5. H. A. Rowland: Bulletin of the American Physical Society 1, 4 (1899)

6. S. Newcomb: Sidereal Messenger 7, 65 (1888)

7. M. Harwit: Cosmic Discovery: the Search, Scope, and Heritage of Astronomy (Basic Books, New York 1981)

8. P. J. E. Peebles, B. Ratra: astro-ph/0207347

9. P. J. E. Peebles: astro-ph/0208037

10. W. Nernst: Verhandlungen der Deutschen Physikalischen Gesellschaft 18, 83 (1916)

11. W. Pauli: 'Die allgemeinen Prinzipien der Wellenmechanik'. In: Handbuch der Physik, Quantentheorie XXIV/1 ed. by H. Geiger and K. Scheel (Springer, Berlin 1933), p. 83

12. F. Wilczek: Physics Today 55, August, p. 10 (2002)

13. R. Penrose: Phys. Rev. Lett. 14, 57 (1965)

14. J. Khoury, B. A. Ovrut, P. J. Steinhardt, N. Turok: Phys. Rev. D 64, 123522 (2001)

15. F. Hoyle, N. C. Wickramasinghe: Astrophys. Space Sci. 268, pp. vii - vii and 1 17 (1999)

16. J. Farley: The Spontaneous Generation Controversy from Descartes to Oparin (The Johns Hopkins University Press, Baltimore 1974)

17. M. J. Crowe: The Extraterrestrial Life Debate, 1750-1900: the Idea of a Plurality of Worlds from Kant to Lowell (Cambridge University Press, Cambridge 1986)

18. Lord Kelvin: Presidential Address to the British Association, Edinburgh (1871) 